\title{
Variations
}

Variations

Revue internationale de théorie critique

$20 \mid 2017$

Expériences oppositionnelles

\section{Prêt de mémoire}

\section{Esteban Lorenzano}

Traducteur : Paola Sedda de l'espagnol et lecture critique de Flavie Prieux

\section{(2) OpenEdition}

\section{Journals}

Édition électronique

URL : http://journals.openedition.org/variations/849

DOI : 10.4000/variations.849

ISSN : 1968-3960

Éditeur

Les amis de Variations

Référence électronique

Esteban Lorenzano, "Prêt de mémoire », Variations [En ligne], 20 | 2017, mis en ligne le 25 avril 2017, consulté le 25 février 2021. URL : http://journals.openedition.org/variations/849 ; DOI : https://doi.org/ 10.4000 /variations.849

Ce document a été généré automatiquement le 25 février 2021.

Les ami•e•s de Variations 


\section{Prêt de mémoire}

\section{Esteban Lorenzano}

Traduction : Paola Sedda de l'espagnol et lecture critique de Flavie Prieux

\section{NOTE DE L'ÉDITEUR}

Ce texte est tiré de l'ouvrage Huellas : voces y trazos de nuestra memoria / Eugenia Azurmendi, Esteban Lorenzano, Martín Elias, Rolando Fernandez, Paula Silva Testa ; ilustrado por María Giuffra. - 1a ed Ciudad Autónoma de Buenos Aires : El Zócalo, 2017. https://cooperativaelzocalo.com.ar/libreria/

\section{I}

1 «Tu dois écrire quelque chose, cubain $^{1} »$, m'écrit Maria sur le tchat, et moi, je continue à y penser, sans trop d'enthousiasme ou, mieux, juste avec l'enthousiasme nécessaire pour continuer à y penser en même temps que je consulte ses dessins et les textes que les autres ont déjà envoyés.

2 La consigne est simple : « écrire quelque chose sur la relation avec ta mère, sur sa vie de militante, sur les années 1970 ». Bon, c'est pas exactement ça mais c'est comme ça que je l'interprète.

3 « Facile ", bien sur. Sauf que je ne sais même pas par où commencer.

4 Néanmoins, il faut bien commencer quelque part. Je crois qu'avec ce truc de la reconstitution j'ai besoin d'aller en arrière, mais pas beaucoup, jusqu'au moment de mes premiers souvenirs, jusqu'au début de l'année 1995, l'époque à laquelle j'ai connu Maria et quelques autres, beaucoup d'entre eux sont après devenus mes amis les plus proches.

5 J'étais rentré en Argentine depuis deux ans, en 1993, et je m'étais baladé par-ci, par-là sans avoir une destination précise, en cherchant une place dans cette ville de Buenos Aires. J'étais tant assoiffé de vie et désireux de faire mon histoire que je ne me rendais 
pas compte à quel point elle était en train de me dévorer, petit à petit, elle m'ajoutait à son histoire et, moi, je me mêlais à elle, je m'endormais dans son murmure confortable.

J'étais dans cet état quand quelqu'un, Lucrecia je crois, un jour me dit: «il y a un groupe de fils des desaparecidos qui sont en train de se réunir, dans les locaux de Familiares ${ }^{2}$, tu n'as pas envie d'y aller? », moi je lui ai dit oui mais je n'y suis pas allé.

7 J'ai attendu un peu, jusqu'au cortège de la résistance de cette année-là, en 1994, où les fils (bah, H.I.J.O.S. ${ }^{3}$, ils venaient tout juste d'ajouter les petits points), ont fait leur apparition, ou bien était-ce plus tard ? En décembre du 1995 ? Je ne sais plus. Ma vie à cette époque-là était tellement intense qu'il me semble que tout ça s'est produit sur plusieurs années alors que ça n'a peut-être duré que quelques mois.

8 Donc, oui, c'est à ce moment que j'ai rejoint le groupe, quand ils étaient déjà là et qu'ils attendaient que d'autres se joignent à eux.

9 Je me rappelle que ce premier jour quand je suis allé à la cave de Riobamba il y avait un groupe plutôt important de filles et de garçons, je ne sais pas dire le nombre exact mais ils étaient assez nombreux. Ils étaient heureux : la manifestation de la résistance les avait fatigués mais également rendus euphoriques. Ils étaient en train de planifier d'autres actions afin de préparer déjà la manifestation du $24^{4}$.

10 Je crois que, dès le début, j'ai précisé que, pour moi, rechercher la reconnaissance de la justice (bourgeoise) c'était bien mais, qu'au fond, cela n'avait pas beaucoup d'importance. Les miettes qu'ils pouvaient nous offrir, magnanimes, ceux qui avaient construit un système auto-proclamé démocratie sur les tombeaux absents d'une génération ne m'intéressaient pas (et je le pense encore aujourd'hui).

11 Obtenir même le plus petit changement semblait une mission impossible.

12 À y penser, la vérité est que nous avions connu défaite sur défaite : la vague de privatisations, la fermeture des usines, le début du chômage structurel et, bien sûr, pour couronner le tout, la " grâce $»^{5}$ pour les oppresseurs.

13 Une défaite sur toute la ligne : le pays avait succombé à la fièvre néolibérale (qui avait déjà débuté pendant la dictature), au consensus de Washington et au découragement généralisé. Dans un tel contexte, s'opposer à « la théorie des deux démons " ${ }^{6}$ semblait une entreprise colossale et, plus encore, d'envisager d'aller au-delà. Ainsi, je suis resté dans ce groupe parce que, tout en ne croyant pas dans la justice bourgeoise, je croyais quand même dans l'importance de la mémoire. Tout était tellement urgent et nécessaire qu'il fallait bien commencer par quelque chose. Pendant ma période à H.I.J.O.S., c'est donc ce que nous avons fait, avec ses bons et ses mauvais côtés. À la fois avec joie et déception, comme tout dans la vie.

\section{II}

14 «Je te le dis sérieusement, che $e^{7}$, tu dois écrire quelque chose». Continue de me dire Maria, et, moi, je continue à ne pas savoir comment répondre à la question qu'elle me pose : «Comment vis-tu cette absence? »

15 En fait, je ne suis pas tellement sûr de l'avoir vécue comme une absence. Je pense à ça et il ne me reste plus qu'à remonter encore plus en arrière, aux années 1980, à l'exil.

16 À ce moment où je grandissais et je commençais à me poser des questions auxquelles personne pouvait répondre. Que faisions-nous dans ce pays étranger ? Pendant 
combien de temps serions-nous restés là ? La question évidente et nécessaire, « Où est maman ?», je ne me la posais pas beaucoup, je crois qu'il était clair pour moi qu'elle n'était plus là et qu'elle n'allait plus y être.

Quand nous sommes arrivés au Mexique personne ne savait réellement ce qui était en train de se passer en Argentine, et encore moins un gamin de 5 ans qui avait été soustrait des mains de la dictature un peu par hasard et un peu grâce à l'obstination de mes grands-parents qui n'ont jamais arrêté de nous chercher, moi et mes frères. Ils ont tout essayé pour nous retrouver, ils nous ont cherchés partout, dans chaque trous où nous pouvions avoir été cachés (même au Campo de Mayo ${ }^{8}$, grâce à un voisin, un ancien colonel qui était parti à la retraite juste à temps pour éviter sa participation à la répression (mais qui n'avait pas non plus dénoncé ce qu'il avait vu), et cela même quand les autres avaient perdu tout espoir.

Je me rappelle qu'une fois mon oncle m'a raconté que le début de la folie de mon père pouvait être daté avec précision, à ce jour du 1977 quand, dans le journal, ils avaient rapporté la nouvelle des «deux terroristes abattus, et, avec eux, deux enfants » et, puisqu'ils ne connaissaient pas encore l'existence de Pablo (le frère cadet), ils avaient pensé qu'il s'agissait de nous. Mon oncle racontait que cette nuit-là, quand il arriva chez lui, mon vieux, en larmes et totalement bourré, avait préparé une veillée funèbre sans corps. Il semble que pour lui tout se termina ce-jour-là, mais il ne serait pas juste de ma part de l'affirmer de manière catégorique.

Je crois qu'il était content de nous voir quand, quelques mois plus tard, ils nous ont retrouvés. Je crois qu'il était content bien que je ne me le rappelle pas comme ça. Tous les souvenirs que j'ai de mon père sont lamentablement amers.

Je me souviens aussi d'un cauchemar récurrent que j'avais dans cette période-là : mon père marchait à la maison sans se rendre compte que quelqu'un d'autre, en tout point semblable à lui mais méchant, le suivait. J'essayais de le prévenir mais il ne m'entendait pas.

21 Au final, le papa méchant balançait mon père par la fenêtre et après il continuait à marcher, en suivant la même routine que mon père mais moi je savais que ce n'était pas lui l'homme qui était en train de marcher. Et pourtant, avec toute cette amertume, cette désolation, cette folie, mon père, à sa manière, a essayé de continuer à lutter, il a essayé de nous transmettre au mieux l'histoire de notre mère.

Pourquoi est-ce que je raconte tout ça?

Parce que, malgré tout, j'ai eu quelque chose dans mon enfance que tout le monde n'a pas forcément eu : la présence constante de ma mère. Savoir parfaitement ce qu'elle faisait et pourquoi elle le faisait, ainsi que la cause pour laquelle elle était morte.

Morte au combat, nous pensions au début, avant la circulation des premières nouvelles à ce sujet, quand, dans la communauté des expatriés, on commençait à parler de desaparecidos.

Qu'est-ce qu'il me reste alors de l'exil ?

27

Il m'a donné une connaissance profonde de mon histoire, enveloppée dans la folie de mon père, l'amour de mes grands-parents et la compagnie de tous ceux qui ont vécu dans ce monde-là et se sont réveillés de la défaite avec la même ingénuité que moi qui n'avais que 12 ans à la fin de la dictature. 
«Oh, che $e^{9}$ arrête de perdre du temps avec ces bêtises et donne-moi ce texte sur ta mère ", insiste, insupportable, Maria. Et, moi, je me rends compte que je suis en train d'écrire sur moi-même et non pas sur ma mère, mais, en même temps, je me demande si cela ne peut pas être fait autrement.

C'est une absence qui s'est transformée il y a quelques temps en un fantôme, ses pas se perdent dans la brume des fantaisies et l'espace qu'il y a entre ce qui s'est passé et ce que nous pensons qu'il s'est passé, s'élargit, se mythifie ou se resignifie, à partir de ce que nous voulons y voir.

Mais bon, toute expérience est vécue subjectivement.

Quoi dire donc de ma mère?

Raquel Rina Menna naquit en Italie et fut, comme de nombreux autres gens, transplantée en Argentine où ses parents émigrèrent à la recherche d'un futur meilleur.

À 16 ans, elle avait tellement cassé les pieds à mes grands-parents qu'ils avaient fini par déménager et transférer l'atelier de couture de Tres Arroyos ${ }^{10}$ à Córdoba ${ }^{11}$ où elle aurait pu fréquenter l'école normale d'instituteurs. Peu de temps après, elle connut et tomba amoureuse de mon père, Luis, qui avait été envoyé de La Plata par le parti ${ }^{12}$ pour créer une structure politique à Córdoba à laquelle venait d'adhérer (entre autres) mon oncle, el Mingo ${ }^{13}$.

34 Je ne sais rien de son histoire d'amour et du mariage qui a succédé mais je sais que mon frère naquit en février du 1969 et, bien évidemment, car ça n'aurait pas pu être autrement, ils l'appelèrent Ernesto. Je naquis trois ans après et mes parents se séparèrent peu de temps après ma naissance. J'ignore les causes de leur rupture mais j'imagine que leurs divergences politiques peuvent avoir joué un rôle : mon père adhéra à l'une des factions de ce qui fut après nommé le G.O.R. ${ }^{14}$, je crois, même si j'en suis pas totalement sûr, et ma mère resta dans le parti où elle continua sa lutte et sa vie (Pablo, mon petit frère, naquit en décembre 1975), jusqu'au moment où elle tomba dans le combat le 29 avril du 1977 à Mercedes ${ }^{15}$, pendant qu'elle défendait la dernière imprimerie qui restait dans les mains du P.R.T. Nous étions là avec elle, tous les trois.

5 Jusqu'ici la vérité est que je ne peux pas en dire beaucoup plus sur cette histoire.

Moi, j'avais 5 ans la dernière fois que je l'ai vue et la dictature m'a enlevé même ce souvenir. J'ai un blocage mental qui m'empêche de me rappeler de n'importe quel événement connecté à ce jour ou aux jours qui l'ont précédé.

7 Bien sûr ils sont passés presque 40 ans depuis et on pourrait penser que c'est là la cause de mon amnésie. Mais le problème est que je me rappelle parfaitement du fait que je n'ai jamais eu ce souvenir. Je me rappelle que mon frère, qui n'est pas non plus trop enclin à parler de cette époque, a dû me prêter ses souvenirs, pour que je n'aie pas ce trou dans mon histoire.

Je sais qu'il a voulu le faire afin que son idée, et non pas forcément son souvenir, puisse me réconforter dans les moments de solitude. À ce point de l'histoire, je serais normalement censé dire quelque chose sur la manière dont j'ai hérité de l'idéologie de ma mère et de ce qu'elle penserait des années qu'elle n'a pas pu vivre. 
Mais cela reviendrait à mentir deux fois.

Premièrement, parce que mon idéologie c'est ma propre responsabilité. Elle peut seulement contenir l'exemple de ma mère (et de mon père aussi), ce qui n'est pas rien, c'est clair, cet exemple est bien là.

Deuxièmement, parce que si je m'identifie à ses idéaux ce n'est pas pour une question d'héritage mais par conviction. Je suis grand et je suis responsable de ce que je pense et de ce que je dis sans devoir attribuer cette responsabilité à ceux qui ne sont plus là.

En ce qui concerne ce qu'elle penserait aujourd'hui, je peux vous en dire encore moins là dessus. Je n'aime pas ceux qui essaient de justifier leurs décisions à travers leurs parents. Et j'aime encore moins ceux qui essaient de se justifier avec des phrases du style « les temps ont changé et même eux aujourd'hui penseraient différemment ».

J'ai aucune idée de ce que ma mère penserait aujourd'hui mais, à partir de ce qu'on dit d'elle et du parti auquel elle adhéra et pour lequel elle lutta jusqu'au dernier instant de sa vie, je ne crois pas qu'elle aurait pu se conformer avec ce mode (ou avec ce pays).

IV

Au final, je ne sais pas si Maria aimera lire ce que j'ai à dire. Ou les autres qui pourraient me lire par la suite. La vérité est que la récupération de la mémoire dans les termes dans lesquels elle est en train de se faire ne m'intéresse pas et ne m'a jamais réellement intéressé. Je comprends la valeur symbolique que certains gestes peuvent avoir ou la valeur réparatrice que de nombreux gens peuvent en tirer. Et pourtant, qu'est-ce que je peux dire de plus ? Pour moi ce n'est pas tellement important que la grâce pour les oppresseurs ait été déclarée inconstitutionnelle. Les procès aux militaires n'ont guère effet sur moi, et leurs condamnations non plus. Et cela non pas car tout ça n'est pas juste ou ne doit pas être fait mais parce que ce n'est pas comme ça que l'on tourne la page dans l'Histoire.

Du moins, pas pour moi.

Oui, ma mère a eut sa lutte et moi j'ai eu (et j'ai) la mienne, marquée par la recherche de la reconnaissance pour les combattants morts dont ma mère fait partie.

Oui, c'est vrai mais ce n'est pas que ça. Selon moi, accepter les termes de cette réconciliation équivaut à renoncer aux causes qui ont provoqué la rupture originelle. Accepter que le mieux que nous puissions faire est cet État, cette société, ce cimetière.

Oui, soit, mais pas comme ça.

Car, il est vrai, nous avons obtenu beaucoup de choses pour lesquelles nous avions lutté dans les années 1990 : annulation de la grâce, réouverture des procès, élargissement et approbation des lois réparatrices; nous sommes en train d'obtenir progressivement des gouttes de justice, parfois avant que les assassins ne meurent de vieillesse, parfois non.

Mais après ? après il y a encore énormément de choses qui manquent, non ?

Il y a encore la police qui a protégé les dictateurs.

Il y a encore les juges qui ont raccommodé leurs lois.

Il y a encore les syndicalistes qui ont livré les camarades.

Il y a encore les partis politiques qui leur ont fourni les fonctionnaires pour préserver l'État. 
Il y a encore les entrepreneurs qui les ont financés.

Et qu'est-ce qu'il n'y a plus alors?

Il n'y a pas la justice pour ceux qui sont morts à cause de la répression policière.

Il n'y a pas Julio Lopez ${ }^{16}$ (et beaucoup d'autres).

Il n'y a pas l'éducation, la santé et le travail pour tous.

Et surtout, il n'y a pas la chose la plus importante, il n'y a pas la liberté : la vraie, la belle, la sociale.

Celle qui peut émerger seulement si nous sommes tous égaux.

\section{NOTES}

1. L'auteur a passé six ans d'exil à Cuba pendant la dictature argentine. Dans ce texte, il mentionne uniquement l'exil au Mexique. L'appellatif «le cubain » se réfère au surnom que les amis et les camarades argentins lui ont attribué quand il est retourné en Argentine.

2. Familiares : Abréviation de "Asociación de Familiares de Detenidos Desaparecidos", est un organisme militant pour la protection des droits humains qui rassemble les familles des victimes de la dictature militaire.

3. H.I.J.O.S. "Hijos por la Identidad y la Justicia, contra el Olvido y el Silencio", est une association impliquée dans la lutte pour les droits humains à laquelle ont adhéré les fils des disparus, les prisonniers politiques, les exilés et les victimes de la dictature.

4. La manifestation du 24 mars a lieu tous les ans et vise à commémorer le début de la dictature militaire (24/03/1976) et à revendiquer le droit à la mémoire et à la justice.

5. «Indulto » dans le texte originel en espagnol.

6. On fait ici référence à des positions qui attribuent une responsabilité égale au terrorisme d'État et à la lutte armée menée par ses opposants dans le déclenchement du conflit politique sanglant qui marqua le pays.

7. «Che » en langue mapuche signifie " gens ». Souvent situé au début ou à la fin de la phrase, il est très utilisé en Argentine pour se référer aux autres dans un langage familier.

8. Campo de Mayo est le quartier général de l'armée argentine ainsi que l'un des principaux centres de détention et de torture clandestins.

9. Voir note $\mathrm{n}^{\circ} 7$.

10. Tres Arroyos est une petite ville d'environ 100.000 habitants située au Sud de Buenos Aires.

11. Córdoba est la capitale de la région éponyme, connue, entre autre, pour la qualité de l'éducation universitaire.

12. El Partido Revolucionario de los Trabajadores - Ejército Revolucionario del Pueblo (PRT-ERP) est une organisation marxiste révolutionnaire qui fut à la tête d'une vaste lutte qui se développa à tous les niveaux de la vie politique et sociale en incluant des formes de guerrilla urbaine et rurale.

13. Domingo Menna, "el Mingo", membre du Comité Central et du bureau politique du PRT-ERP, fut l'un des responsables de l'organisation du parti et de différentes sections syndicales (notamment celle de la ville Villa Constitución); il prit partie à l'évasion du prisonnier Rawson en 
1972. Il fut déclaré disparu à partir du 19 juillet 1976 dans le cadre de la même opération qui provoqua la mort de Santucho (le principal dirigeant du parti) et de Urteaga.

14. GOR - El Grupo Obrero Revolucionario - fut une fraction du PRT-ERP. La différence principale entre les deux groupes se situait au niveau du caractère de la lutte révolutionnaire : tandis que le PRT-ERP soutenait la nécessité de créer une armée capable d'affronter la dictature militaire, le GOR affirmait que la lutte armée aurait dû être subordonnée au développement de la lutte du prolétariat et des masses. Le développement de ce dernier fut en effet fondamental pour la mobilisation des quartiers ouvriers et son action fut en général liée au contexte des usines et des conflits syndicaux.

15. Mercedes est une localité située aux alentours de Buenos Aires.

16. Jorge Julio Lopez fut le principal témoin dans le procès contre l'oppresseur Miguel Etchecolatz pendant lequel il raconta les modalités de sa séquestration et les tortures subies. Il disparut le 18 septembre 2006 en plein régime démocratique. L'identité de son ravisseur reste encore aujourd'hui inconnue. Pour les militants des droits humains, l'expérience de Lopez démontre la permanence des structures répressives.

INDEX

Mots-clés : dictature argentine, desaparecidos, lutte armée, engagement, droits humains

\section{AUTEURS}

\section{ESTEBAN LORENZANO}

Un guevariste 